\title{
Texture Retrieval based on a Non-parametric Measure for Multivariate Distributions
}

\author{
Vasileios K. Pothos \\ Electronics Laboratory, \\ Dept. of Physics, \\ University of Patras, \\ Patras 26500, Greece \\ +302610997445 \\ bpothos@upatras.gr
}

\author{
Christos Theoharatos \\ Electronics Laboratory, \\ Dept. of Physics, University \\ of Patras, Patras 26500, \\ Greece \\ +302610 997445 \\ htheohar@upatras.gr
}

\author{
George Economou \\ Electronics Laboratory, \\ Dept. of Physics, \\ University of Patras, \\ Patras 26500, Greece \\ +302610997445 \\ economou@physics. \\ upatras .gr
}

\author{
Apostolos Ifantis \\ Control System and Signal \\ Laboratory, Dept. of \\ Electrical Engineering, \\ Technological Institute of \\ Patras, Patras 26334, \\ Greece. +30 2610369254 \\ ifantisa@teipat.gr
}

\begin{abstract}
In the present study, an efficient strategy for retrieving texture images from large texture databases is introduced and studied within a distributional-statistical framework. Our approach incorporates the multivariate Wald-Wolfowitz test (WW-test), a non-parametric statistical test that measures the similarity between two different sets of multivariate data, which is utilized here for comparing texture distributions. By summarizing the texture information using standard feature extraction methodologies, the similarity measure provides a comprehensive estimate of the match between different images based on graph theory. The proposed "distributional metric" is shown to handle efficiently the texture space dimensionality and the limited sample size drawn from a given image. The experimental results, from the application on a typical texture database, clearly demonstrate the effectiveness of our approach over other texture distribution (dis)similarity metrics. In addition, its performance is used to evaluate several approaches for texture representation. Even though the classification results are obtained on grayscale images, a direct extension to color-based ones can be straightforward.
\end{abstract}

\section{Categories and Subject Descriptors}

H.3.3: [Information Storage and Retrieval]: Information Search and Retrieval - Information filtering, Retrieval models, Search process, Selection process.

I.5.3 [Pattern Recognition]: Clustering - similarity measures.

\section{General Terms}

Algorithms, Measurement, Performance.

\section{Keywords}

Texture retrieval, Non-parametric distance, distributional metric, statistical graph matching, texture features.

\footnotetext{
Permission to make digital or hard copies of all or part of this work for personal or classroom use is granted without fee provided that copies are not made or distributed for profit or commercial advantage and that copies bear this notice and the full citation on the first page. To copy otherwise, to republish, to post on servers or to redistribute to lists, requires prior specific permission and/or a fee.

CIVR'07, July 9-11, 2007, Amsterdam, The Netherlands.

Copyright 2007 ACM 978-1-59593-733-9/07/0007 ...\$5.00.
}

\section{INTRODUCTION}

Texture is an important visual property of the materials, and together with color and shape are fundamental issues encountered in many, low level, image analysis and computer vision tasks. The study of texture alone is recognized to be a difficult subject in image science and among the different research directions pursued in the field. The basic building elements that constitute a texture retrieval system are (i) robust texture representation and (ii) design of a (dis)similarity measure between texture distributions.

Texture representation is known to be a difficult problem due to the high - and usually unknown - true dimensionality of the feature space required to represent properly the useful information. Texture, as opposed to color, is an area property and not a point one and is characterized by features like roughness, variability, repeatability, directionality etc, which are defined over a certain spatial extent. Many methods for texture retrieval and classification use the energy distribution in the frequency domain to identify texture. Classical examples include (but are not limited to) image decomposition by filtering with a subband or wavelet filter bank [1-4] and appliance of a linear transformation by a Fourier or discrete cosine transform (DCT) [5-7]. In those methods, texture can be modelled by the fusion of marginal densities of subband image coefficients. Following this approach, one can extract samples from the texture distribution by utilizing small neighbourhoods of scale-to-scale coefficients. Components of the multivariate texture-distributional vectors are formed using the spatially localized coefficients, at different image decomposition levels.

The other key parameter of a texture retrieval system is the definition of a dissimilarity measure between different textures. Towards this objective, several methods have been proposed based on histogram comparison (e.g. see [8]). However, these techniques exhibit several drawbacks. The necessary trade-off during the binning procedure has been recognized as the major cause of this limitation, and as dimensionality increases, the histogram-based measures fail to perform effectively [9]. An alternative approach to measure texture resemblance is by means of non-parametric statistical tests that make no parametric assumptions about the underlying sample distribution $[7,8,10]$. This guarantees the similarities to be assessable in terms of statistical significance, but avoids direct statistical parameter estimation. Non-parametric distributional-based methods share a common characteristic; they require the availability of a number 
of (identically-distributed) independent samples from the underlying distribution to operate on. These samples need to be extracted from the available data. In the core of the proposed technique lies a non-parametric test dealing with the "Multivariate Two-Sample Problem" [11], which has been adopted here for expressing texture image similarity. The specific test is a multivariate extension of the classical Wald-Wolfowitz test (WWtest) and compares two different samples of vectorial observations (i.e. two sets of points in $\mathbf{R}^{\mathbf{P}}$ ) by checking whether they form different branches in the overall minimal spanning tree (MST) [12]. The output of this test can be expressed as the probability that the two point-samples are coming from the same distribution. Its great advantage is that no a-priori assumption about the distribution of points in the two samples is a prerequisite.

The success of the previously described methodologies for feature extraction and distributional similarity estimation are tested on part of the OUTex texture database. The retrieval problem is stated as follows; given a new texture sample, find from a predetermined set of texture samples those that contain the same texture. Results should be in accordance with the intuitive notion of visual similarity of the different textures. Special effort is taken to judge all techniques under equal terms and use the available database in an optimal way. Regarding texture feature extraction techniques, three different methodologies are incorporated in this study, which are considered as golden standard in the scientific community: wavelet transform, DCT and Gabor filters. We also present retrieval results directly in the image domain, by straightforwardly applying the multivariate WW-test without initiating any indexing methodology.

The rest of the paper is organized as follows. In Section 2, the multivariate WW-test is shortly presented. Section 3 briefly covers the theoretical aspects of the utilized methodologies for texture feature extraction. The experimental evaluation using a typical greyscale image database is presented in Section 4, including a short discussion on the experimental observations. Finally, conclusions are drawn in Section 5, along with an outline of our future research objectives.

\section{THE MULTIVARIATE WALD - WOLFOWITZ TEST (WW-TEST)}

A non-parametric test dealing with the "Multivariate Two-Sample Problem" [11] has been adopted in the proposed approach for estimating texture content similarity in a reliable and convenient way. The utilized test is a multivariate extension of the classical statistical test of Wald and Wolfowitz and compares two different samples of vectorial observations. The output of the test can be expressed as the probability that two point-samples are coming from the same distribution. Its great advantage is that it is modelfree and this stems from the graph-theoretic origin of the test, which is actually based on the concept of MST-graph [12].

In the multivariate WW-test, the graph is built over points in $\mathbf{R}^{\mathbf{p}}$ : a single node corresponds to every given point, the weight associated with every possible edge is the corresponding interpoint Euclidean distance, while the edges involved in the construction of MST are the ensemble of straight-line segments connecting all points with minimum total length. WW-test can be used to test the hypothesis $\mathbf{H}_{\mathbf{0}}$, whether any two given multidimensional point samples $\left\{X_{i}\right\}_{i=1: m}$ and $\left\{Y_{i}\right\}_{i=1: n}$ are coming from the same multivariate distribution. At first, the two data samples of size $m$ and $n$ are considered, respectively, from distributions defined in $\mathbf{R}^{\mathbf{P}}$. Then, the sample identity of each point is not encountered and the MST of the overall sample is constructed. Based on the sample identities of the points, a test statistic $R$ is computed. $R$ is the total number of runs, while a run is defined as a consecutive sequence of identical sample identities and can be defined as the number of disjoint subtrees that finally result. Rejection of $\mathbf{H}_{\mathbf{0}}$ is for small values of $R$. The null distribution of this statistic is derived based on combinatorial analysis [11]. It has been shown that the quantity:

$$
W=\frac{R-E[R]}{\sqrt{\operatorname{Var}[R \mid C]}}
$$

approaches (asymptotically) the standard normal distribution, while the $E[R]$ and $\operatorname{Var}[R \mid C]$ of $R$ depend on the sizes $m$ and $n$ of the two point-samples and can be computed as:

$$
\begin{aligned}
& E[R]=\frac{2 m n}{N}+1, \text { and } \operatorname{Var}[R \mid C]=\frac{2 m n}{N(N-1)} \times \\
& \times\left\{\frac{2 m n-N}{N}+\frac{C-N+2}{(N-2)(N-3)}[N(N-1)-4 m n+2]\right\}
\end{aligned}
$$

where $N=m+n$ and $C$ is the number of edge pairs sharing a common node. Its importance is that using simple formulae, the significance level (and p-value) for the acceptance of the hypothesis $\mathbf{H}_{\mathbf{0}}$ can be readily estimated.

In the present work, the above test is utilized as follows. Given the feature extraction step (analysed in the following Section), a representative set of textural characteristics - formed as feature vectors - is selected for a couple of texture images that are going to be compared. $W$ is then computed and used as a similarity measure in a way that the more positive its value is, the more similar the two images are [13]. The $W$-quantity computed between pairs of images plays the role of a "distributional distance” acting on samples of image constituents, and therefore inherits interesting invariant characteristics such as rotation and translation invariance. Under this perspective, the WW-test can be directly incorporated, for example, in classification and retrieval processes from image databases, with the great advantage of being suitable for dealing with multivariate distributions.

\section{METHODOLOGIES FOR TEXTURAL FEATURE EXTRACTION}

\subsection{Texture extraction using Wavelets}

In order to accomplish indexing and retrieval practices, a compact representation of image texture needs to be derived in the transform domain [4]. In the general case, the wavelet transform is applied to a given image in $N$ decomposition levels, decomposing each level into four independent and spatially oriented channels [14]. In this way, the subbands $L L, L H, H L$ and $H H$ are resulted. Among them, the subbands $L H$ and $H L$ are mixed via the type:

$$
L H H L_{n}=\sqrt{L H_{n}^{2}+H L_{n}^{2}}, n=1,2, \ldots, N
$$

in order to produce the $L H H L$ subband, for each decomposition level $n$. 


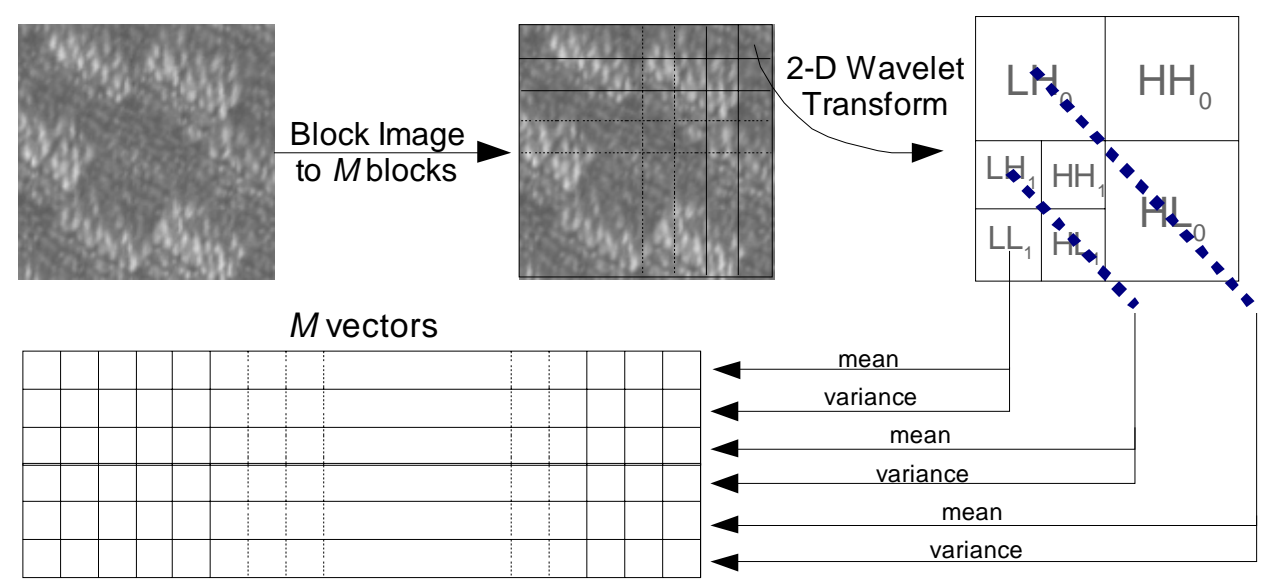

Figure 1. Construction of the wavelet feature vectors to describe texture information.

To be in agreement with the other feature extraction methodologies (that will be presented in the following subsections), we originally partitioned the image into $M=64$ nonoverlapping square blocks and, afterwards, applied the wavelet decomposition to each one of them as introduced above. The $L L_{N}$ and the $L H H L_{n}$ subbands, which are $K=N+1$ in total, can now be utilized for texture feature extraction using standard statistical techniques. In our study, we used $N=2$ decomposition levels due to the small size of the images blocks. For each subband, we calculated the mean $\mu_{[k, m]}$ and the variance $\sigma_{[k, m]}$ of the energy distribution of the transform coefficients using the following equations:

$$
\begin{gathered}
\mu_{[k, m]}=\iint\left|W_{[k, m]}(x, y)\right| d x d y, k=1,2, \ldots, K \\
\sigma_{[k, m]}=\sqrt{\iint\left(\left|W_{[k, m]}(x, y)\right|-\mu_{[k, m]}\right)^{2} d x d y}, m=1,2, \ldots, M
\end{gathered}
$$

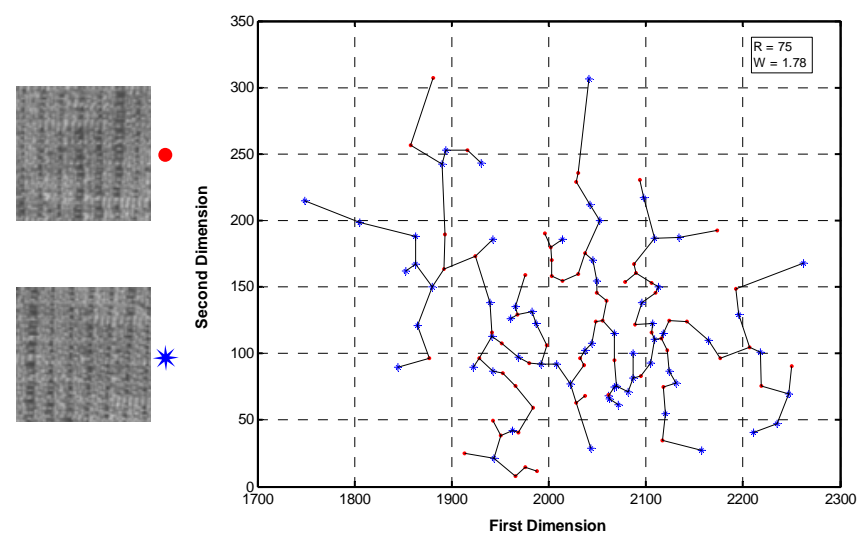

Figure 2. WW-test in 2-D for a pair of similar textured images (labelled by “o” and “*” for each distinct image), using input vectors extracted based on the wavelet transform. where $W_{[k, m]}$ corresponds to $L L_{1}, L H H L_{1}$ or $L H H L_{0}$ of the $m$ - th block. Based on the above procedure we can create a set of $M$ - vectors in the $6-D$ that best describe each texture image. A representative scheme that sketches the above procedure is illustrated in Fig. 1. The extracted texture features, can be used as input vectors to the $\mathrm{WW}$-engine to compare two texture images.

Fig. 2 exemplifies the performance of $\mathrm{WW}$-test for a pair of similar textured images. In this panel, different labels are associated with each of the two images to be compared. Based on the image they are coming from, the extracted vectors are labeled accordingly using a "star" and a "dot" symbol. By contrasting the overall MSTs, it becomes evident that in the case of similar images there are many edges having differently labeled nodes as endpoints, since the selected vectors tend to mix together. More specifically, there are 74 edges ending on nodes coming from different images and therefore splitting the overall MST into 75 subgraphs, thus $R=75$ (and $W=1.78$ ).

\subsection{Texture extraction using DCT}

The Discrete Cosine Transform (DCT) has been widely used in the literature for efficient texture feature selection. It uses cosines of varying spatial frequencies as basis functions and is commonly known for its implementation in the JPEG compression standard $[5,15]$. In order to extract textural attributes, the images are initially partitioned into $N \times N$ pixel-blocks, with $N=16$ in our case. The block size was selected in order to reduce the number of extracted feature vectors and also try to effectively capture the texture information using a larger image patch. In addition, it was experimentally verified to produce enhanced retrieval results compared to a smaller (e.g., $N=8$ ) pixel-block.

The DCT is afterwards applied to each distinct block, as illustrated in Fig. 3. From each DCT block, texture can be now represented by a feature vector $V_{m}$, with $m \in[1,2 N-2]$, the elements of which are the square sums of coefficients of the corresponding diagonals (i.e., zig-zag traversal lines) [7], as shown in Fig. 3. The vector resulting from the zig-zag ordering contain all the AC coefficients starting from the upper left location (i.e., $(0,1))$ to the bottom right (i.e., $(N-1, N-1)$ ). 


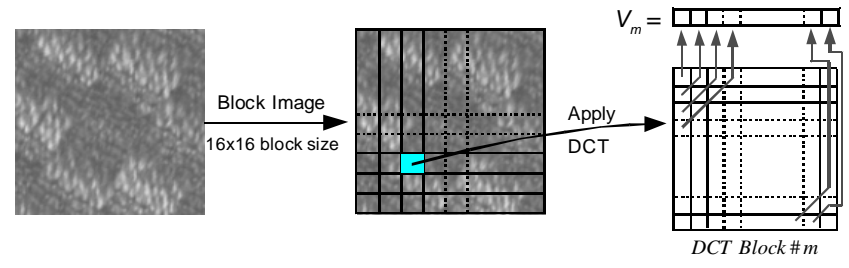

Figure 3. Construction of the DCT feature vectors to describe texture information.

Assuming that a given image is initially divided into $M$ blocks of $16 \times 16$ pixels, then a set of $M$ feature vectors can be extracted that best describes the texture image content of the particular image. The specific indexing scheme was found to be robust, when similarity-based image rotation is encountered [7].

Following the same principle as in Section 3.1, the DCT-based texture features are used as input vectors to the WW-engine for multivariate texture-image matching. In Fig. 4, an example is illustrated for a pair of dissimilar - this time - textured images. Even though we are operating in a multidimensional space, the specific example is conceptualized in a 2-D domain for visualization feasibility, using also different symbol for endpoints coming from different images. By constructing the MST, in the case of dissimilar images the extracted vectors are clearly separated from each other, forming clusters at different locations in the corresponding feature space. Therefore, there are only a few edges (i.e., 16) having endpoints coming from different images, spanning the overall graph into 17 subgraphs, thus $R=17$ (and $W=-8.54$ ).

\subsection{Texture extraction using Gabor Filters}

The relation between the human vision system and the Gabor filters is a strong motive to test Gabor filtering for texture feature extraction [16]. Spatially, a Gabor function is a Gaussian modulated sinusoid. In his work, Daugman $[17,18]$ generalized the Gabor function in 2-D:

$$
G(x, y)=\frac{1}{2 \pi \sigma_{x} \sigma_{y}} e^{-\pi\left[\frac{\left(x-x_{0}\right)^{2}}{\sigma_{x}{ }^{2}}+\frac{\left(y-y_{0}\right)^{2}}{\sigma_{y}{ }^{2}}\right]} e^{i\left(\xi_{0} x+v_{0} y\right)},
$$

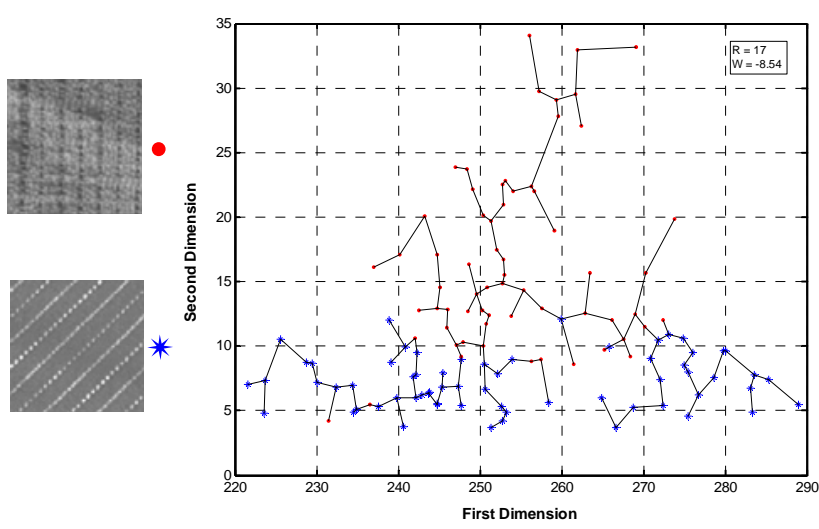

Figure 4. WW-test in 2-D for a pair of dissimilar textured images (labelled by “o” and “*” for each distinct image), using input vectors extracted based on the DCT transform.

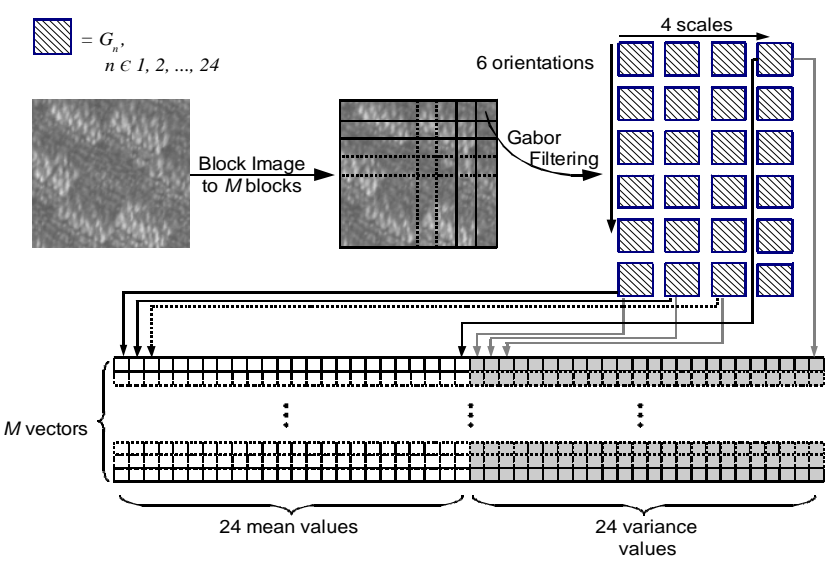

Figure 5. Construction of the Gabor feature vectors to describe texture information.

where $\left(x_{0}, y_{0}\right)$ is the center of the field in the spatial domain and $\left(\xi_{0}, v_{0}\right)$ is the optimal spatial frequency of the filter in frequency domain. $\sigma_{x}$ and $\sigma_{y}$ are the standard deviations of the elliptical Gaussian for axis $x$ and axis $y$ respectively.

In order to extract texture information, we firstly partition the texture images into $M$ non-overlapping rectangular blocks. Then, the Gabor filters are applied using four different scales and six different orientations, creating $N=24$ new filtered subimages. The resulted filtered subimages are obtained by computing the magnitude from the real $G_{\Re_{n}}$ and imaginary $G_{\Im_{n}}$ parts of each n subband of the Gabor filters:

$$
G_{n}=\sqrt{G_{\mathfrak{R}_{n}}^{2}+G_{\mathfrak{\Im}_{n}}^{2}}, n=1,2, \ldots, N
$$

where $G_{n}$ substitutes $W_{[k, m]}$ in (3) and (4), which are used to calculate the mean and variance for each one of the filtered subimages.

In [19], a $2 \times N-D$ multidimensional vector is constructed so as to be used for similarity matching using a valid (dis)similarity measure (i.e., the sum of Euclidean distances). In our study, a $2 \times 24$ dimensional feature vector is built for the description of texture information, corresponding to the mean and variance values per filtered subimages that are contained in each corresponding block. In the final stage, a total number of $M$ feature vectors of $48-D$ is constructed for the description of the texture information of all database images, which are utilized in the multidimensional WW-test for similarity matching. A representative scheme of the previously reported technique is illustrated in Fig. 5, clearly sketching the overall procedure.

\section{EXPERIMENTAL SET-UP}

\subsection{Texture database - performance measures}

In order to test the performance of the proposed non-parametric distance-based classification techniques on texture images, using the texture features discussed in Section 3, the OUTex database (University of Oulu Texture database) was used in our experiments. The utilized dataset contains 24 distinct texture 


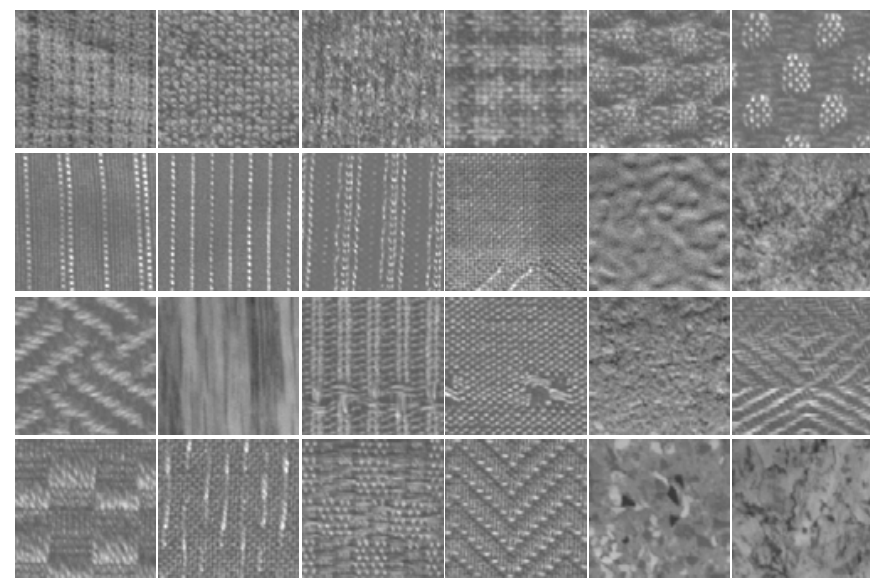

Figure 6. Illustrative examples of the 24 categories that constitute the utilized texture database

categories, having 180 greyscale images of similar texture of size in each class and thus resulting in a total number of 4320 texture images of size $128 \times 128$ pixels. The amount of images comprising a single category is formed by using seven different texture orientations per twenty alike texture images. In Fig. 6, 24 sample images are presented, one for each texture category.

The most widely used performance measures for testing the efficiency and accuracy in information retrieval are the two quantities precision (Pr) and recall ( $\mathrm{Re}$ ) [20], which in our case were used as follows. For a given query image, let $S$ be the number of similar images that constitute a specific category in the image database ( $S=180$ as mentioned before) and $T$ be the set of total retrieved results (a user-defined number controlling the size of the selected list of images returned after querying by example, e.g., the top 10 images). If $Z$ is the number of retrieved images coming from the same class (i.e., correct results) among the $T$ images in the selected list, then $\operatorname{Pr}$ and Re are defined as [20]:

$$
\operatorname{Pr}=\frac{Z}{T} \quad \text { and } \quad \operatorname{Re}=\frac{Z}{S}
$$

In our experiments, 216 query images were used, which were formed by randomly selecting 9 images of similar texture for each distinct class. For comparing the efficiency of the multivariate WW-test, the quantized histogram was utilized for representing the texture distribution of each database image, along with three different (dis)similarity measures that belong to a specific distance category [8]. In this way, histogram intersection (HI) [21] was selected from the heuristic histogram distance category [8], and Kullback-Leibler Divergence (KLD) and JeffreyDivergence (JD) from information-theory divergencies [4, 8]. In addition, the $L_{1}$ - norm was also incorporated in our experimental comparisons, which has been proven to perform well in the case of the Gabor filtering technique [22]. The specific metric is directly applied to compare single multidimensional vectors $\bar{f}=\left[f_{1} f_{2} \ldots f_{N}\right]$, and is provided by the formula:

$$
d\left(\overline{f_{1}}, \overline{f_{2}}\right)=\sum_{m=1}^{N}\left|f_{1 m}-f_{2 m}\right|
$$

In order to create single multidimensional vectors from the ensemble of vectors that are selected using each particular feature extraction technique, we simply compute the mean value from all set of vectors in each dimension. Alternatively speaking, the vector $\bar{f}$ is bound to represent the mass-centroid of the distribution under study.

\subsection{Experimental results}

In what follows, all textural feature extraction methodologies (presented in Section 3) are exemplified using the multivariate WW-test as our standard (dis)similarity measure, which in turn is compared against all the other - previously reported (dis)similarity measures. The reason for constructing the experimental tests is two-fold: on the one hand our goal is to validate the performance of the proposed non-parametric statistical test, in comparison to other approaches that are considered classical in the image retrieval community; on the other hand we aim to estimate the effectiveness of the different feature extraction methodologies for building-up a textural image retrieval scheme.

The retrieval results that compare the feature extraction methodologies with the different (dis)similarity measures are summarized in Fig. 7. In the case of the wavelet method (described in Section 3.1), the output of the proposed technique for texture feature matching is depicted in Fig. 7(a). Evidently, the utilized WW-engine outperforms all other (dis)similarity measures, yielding quite good retrieval rates. In the specific example, two decomposition levels were used in the implementation of the wavelet transform, producing a 6-D texture feature space. In addition, Fig. 7(b) present the computed results coming from the DCT technique (introduced in Section 3.2). In this case, the precision is also substantially higher from all other comparison histogram methods, with the WW-test yielding the best results and the $L_{1}$ distance having $\sim 6 \%$ lower precision than the proposed statistical measure. The higher precesion accuracy is provided using Gabor filters in the feature extraction stage (presented in Section 3.3), as depicted in Fig. 7(c). Using the WW-test for comparing the multivariate texture distributions, we achieved an average precision of more than $95 \%$ for the entire database. The specific effectiveness can safely guide the built-up of an image retrieval scheme for texture datasets, based on Gabor filters (for image-indexing) and the multivariate WW-test (for image-matching, i.e., using pairwise comparisons of texture distributions).

It has to be noted that although $\mathrm{HI}$ and $L_{1}$ distances are closely related there is a big performance difference. In this work $\mathrm{HI}$ is approximately calculated as the mean value of $\mathrm{HI}$ distances between the corresponding pairs of vectors of the two distributions. Instead we compute $L_{1}$ over the mass-centroids of the two distributions. The good performance of the $L_{1}$ can easily be explained, as similar textures have similar frequency characteristics in their segments (blocks), thus the use of the mean value over them produce closely mass-centroids. The other measurements instead are used over the distributions and not their mass-centroids, producing comparative lower performance.

Finally, all (dis)similarity measures were compared directly in the image domain, without the involvement of any feature extraction 


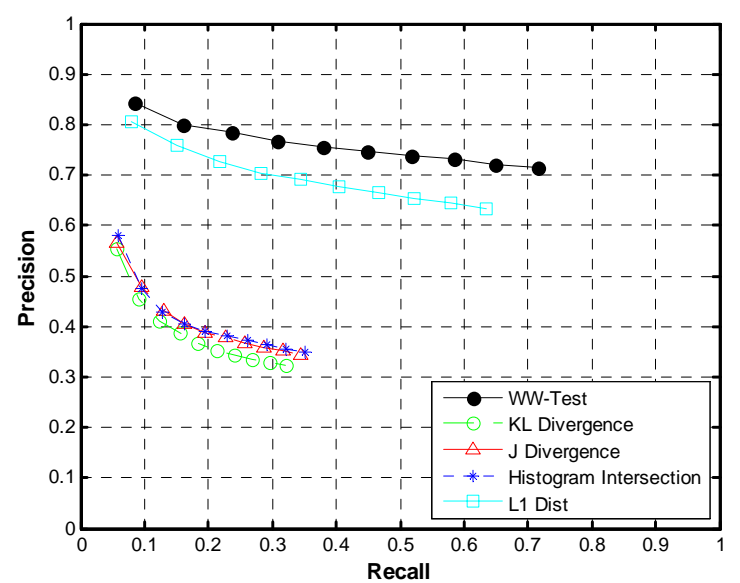

(a)

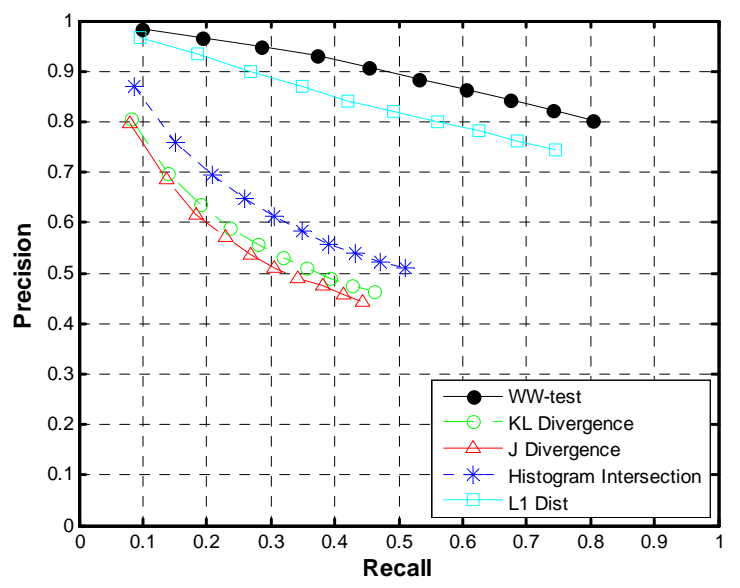

(c)

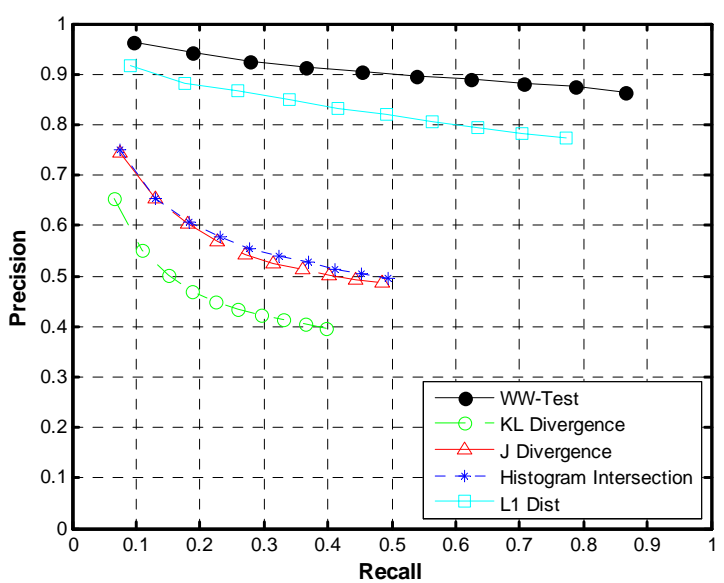

(b)

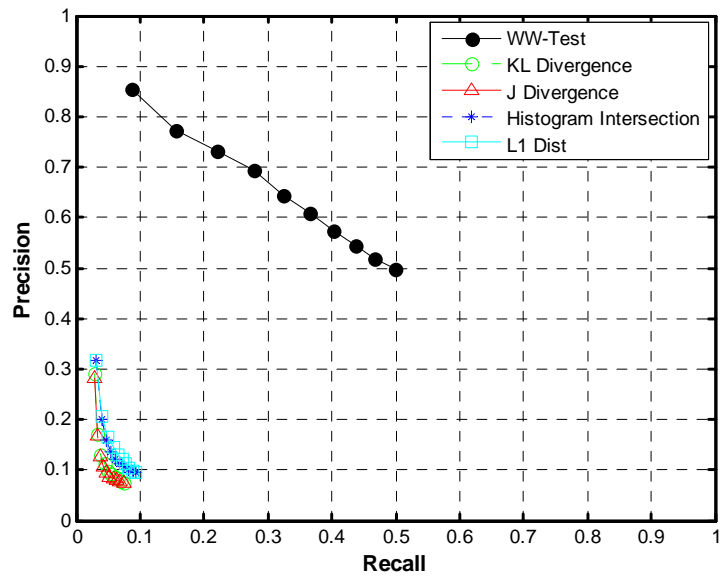

(d)

Figure 7. Precision vs Recall results of the four methodologies presented in Section 3, using other (dis)similarity measures. (a) using wavelets, (b) using DCT, (c) using Gabor filters and (d) in the image domain.

technique. This was dictaded by the need to estimate the performance of the involved pattern matching in a straightforward way, before applying any pre-filtering scheme that disassociates the frequency energy inside each texture image. For this reason, each texture image was first divided into $8 \times 8=64$ image-blocks, having a total of $16 \times 16=256$ pixels each (since all database images are comprised of $128 \times 128$ pixels). Then, a multidimensional vector was constructed by concatenating the pixels involved inside each image-block, building in this way a $256-D$ texture-feature vector. By representing the grayscale values of all image-blocks, 64 vectors were computed, lying in a 256-D feature space. Thus, image similarity between two texture images can be estimated by computing the similarity of their vector distributions, directly in a multidimensional feature space. By comparing all the introduced measures using the predefined technique, interesting results are revealed in Fig. 7(d). As we can perceive, histogram-based measures, as well as the $L_{1}$ distance, fail to meet the requirements induced by the construction of the multidimensional feature space. Their overall performance is pretty discouraging. On the other hand, the multivariate nature of the $\mathrm{WW}$-test makes it an appropriate gauge for texture feature matching, where high-dimensionalities are involved.

By comparing all different aspects of the involved study under the experimental umbrella of Fig. 7, the following observations/ notices can be made. The involved technique using Gabor filtering over image blocks presented in Section 3.3 provides the response with the higher precision. In addition, the results coming from the image domain approach are the most interesting ones. In 256 dimensions, WW-test can be successfully used as a similarity measure yielding a precision of $77 \%$ for the $T=10$ images of the selected list. On the contrary, all other histogram techniques plus the $L_{1}$ metric which performed quite close to the proposed measure in all previous cases, result in an extremely low precision index. Due to the high number of bins (256 in our experiments) and the relatively small number of values that can be taken (i.e. 256 greyscale values), the computed histograms are very sparse and relatively empty. This leads to erroneous matches when used as distributional distance estimates. Moreover, the $L_{1}$ distance fails to perform too, however, not because of the high dimensions 


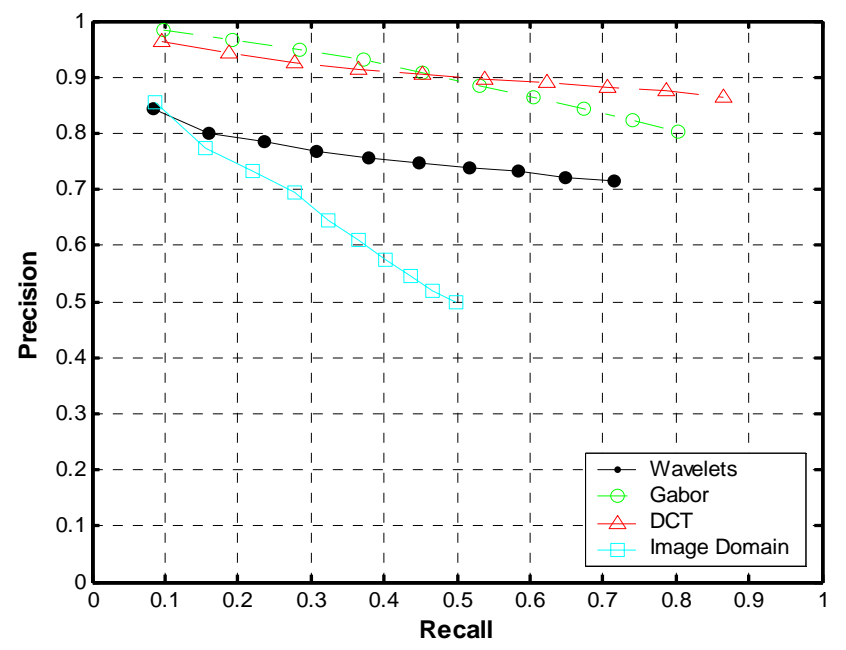

Figure 8. Summarization of the classification error rate results produced using the four methodologies treated at section 3, using the WW-Test as similarity measure.

of the feature space, but rather due to the uncompressed nature of the involved data. Owing to the arbitrary position (or shape) of the texture in the image plane, the vectors coming from each image-block are placed in an almost random location, even in the case of the same kind of texture. By averaging the 64 extracted multidimensional vectors to one central vector in order to be inserted in the $L_{1}$-norm, that structure of the feature space is lost, resulting in the consequent failure of the involved distance metric. However, this did not occur in all the other feature extraction methodologies, since the induced data-compression of the energy frequencies did not alter the hidden structure of the texture image that characterized the texture. On the other hand, the multivariate nature of the WW-test and more specifically the construction of the MST retains the texture structure of the arbitrary vectors. Graph theory, and more specifically the MSTgraph, puts emphasis on the structural relationships between the extracted characteristics. In addition, the MST provides a compact description of a point set and characterized by determinacy [23].

The precision vs. recall results for all distinct methodologies presented in Section 3, using the non-parametric WW-test as the similarity measure for pairwise comparisons, are illustrated in Fig. 8. It can be clearly noticed that the introduced distributionalbased statistical measure works better in the case of the Gabor filters, as well as the DCT-based approach. It should be mentioned that the selected window size for texture analysis in the utilized database are influenced both by the size of the available images and the texture variation included in each image. The very good results obtained using Gabor filters are partially attributed to the particular construction of the texture database. Notice that, different orientations of almost the same texture were included and the directional selectivity that these filters possess is well known. Nevertheless it is observed that Gabor filters together with the DCT decompose a texture image into its subbands in a more robust way.

\section{DISCUSSION}

In the present study, a novel approach is presented to tackle the texture retrieval problem. The multivariate statistical WW-test, which is based on the construction of an MST, is utilized here to estimate the gauge between two distinct distributions. Its generic character stems from the fact that by altering the character of texture image characteristics, we can modify the flavour of formulated queries. The multidimensional test can adequately operate even with a small number of distributional samples and is well suited for texture matching. Part of the flexibility of our proposal is due to the statistical nature of the core procedure, the WW-test, and specifically its multivariate foundation. Not only different image characteristics can - in principle - be combined naturally in one type of query, but also different types of queries can evolve independently and their results can be compared across types. The latter is a direct consequence of the fact that the measured W-index relates directly to significance-level and therefore can be used as an absolute measure to rank among the results of different types of query [13].

It is worthy to note that the computational cost needed for the creation of the MST using Kruskal algorithm is of order $O(N \log N)$, where $N$ is the number of vectors, which grant WW-Test the advantage of low computational cost for relative small $N$; in our case $N=128$. In addition, the number of space dimensions has a slight impact on the computational cost.

In order to extract textural characteristics from a set of given images, individual texture samples were extracted from the images of the OUTex texture database by partitioning the image into non-overlapping regions of almost homogenous texture content. The intrinsic dimensionality of the texture regions was computed by means of image decomposition, implementing some of the well-established techniques. The efficiency in textural feature extraction of the different methods, as well as the competence of the above measure in distributional texture image representations, was tested with quite satisfactory results, which yield future ideas for research and application.

As a scheduled extension of our work, we can straightforwardly implement the application of the introduced methodology to color or multispectral images for texture retrieval and/or classification tasks. This can be accomplished by placing other subband's texture information to higher dimensions in the feature space. However, special care has to be taken to the number of extracted feature vectors in the case of multispectral images, due to the "curse of dimensionality" [24]. In addition, more has to be done in order to overcome the problem that arises from the weakness to capture texture patterns that have different plain scales. This might be accomplished by utilizing adaptive scalable blocks, by means of - each time - different sized block patches inside the input images. For example, the usage of representative samples (instead of simple random patches) can be adopted, by using a vector quantization technique like the "Neural-Gas" algorithm $[25,26]$. In this way, the texture information can be efficiently captured in different scales, making use of possible regions of interest. Moreover, texture segmentation algorithms could be incorporated towards this solution, among the plethora that is available in the literature the recent years (e.g., [27-30]). Finally, the use of alternative ways to extract texture information based on high-level features (i.e., semantic-based texture attributes), so as to correlate better with the human perceptual inspection, is of 
crucial importance [31], as well as the potential application of combining other primitives in the feature extraction methodology.

\section{ACKNOWLEDGMENTS}

This work was financed by the European Social Fund (ESF), Operational Program for Educational and Vocational Training II (EPEAEK II), and particularly the Program "New graduate programs of University of Patras”.

\section{REFERENCES}

[1] Randen T., and Husoy J.H. Filtering for Texture Classification: A Comparative Study. IEEE Trans Pattern Anal Mach Intell, 21, 4 (1999), 291-310.

[2] Leung T., and Malik J. Representing and Recognizing the Visual Appearance of Materials using Three-dimensional Textons. Int J Comput Vis, 43, 1 (2001), 29-44.

[3] Laine A., and Fan J. Texture classification by wavelet packet signatures. IEEE Trans Pattern Anal Mach Intell 15, 11 (1993), 1186-1190.

[4] Do M.N., and Vetterli M. Wavelet-based texture retrieval using generalized Gaussian density and Kullback-Leibler distance. IEEE Trans Image Process 11, 2 (2002), 146-158.

[5] Mandal M.K., Idris F., and Panchanathan S. A critical evaluation of image and video indexing techniques in the compressed domain. Image Vision Comput, 17, 7 (1999), 513-529.

[6] Zhong Y., and Jain A.K. Object localization using color, texture and shape. Pattern Recognit 33, (2000), 671-684.

[7] Theoharatos C., Pothos V.K., Laskaris N.A., Economou G., and Fotopoulos S. Multivariate Image Similarity in the Compressed Domain using Statistical Graph Matching. Pattern Recognit, 39, 10 (2006), 1892-1904.

[8] Rubner Y., Puzicha J., Tomasi C., and Buhmann J.M. Empirical evaluation of dissimilarity measures for color and texture, Comput. Vision Image Understand 84, (2001), 2543.

[9] Leow W.K., and Li R. The analysis and applications of adaptive-binning color histograms. Comp Vis Im Und 94, 1-3 (2004), 67-91.

[10] Ojala T., Pietikainen M., and Harwood D. A comparative study of texture measures with classification based feature distributions. Pattern Recognit 29, 1 (1996), 51-59.

[11] Friedman J.H., and Rafsky L.C. Multivariate Generalizations of the Wald-Wolfowitz and Smirnov Two-Sample Tests. Ann Statist, 7, 4 (1979), 697-717.

[12] Zahn C.T. Graph-Theoretical Methods for Detecting and Describing Gestalt Clusters. IEEE Trans Comput, C-20, 1 (1971), 20-68.

[13] Theoharatos C., Laskaris N.A., Economou G., and Fotopoulos S. A Generic Scheme for Color Image Retrieval based on the Multivariate Wald-Wolfowitz test. IEEE Trans Knowl Data Eng 17, 6 (2005), 808-819.

[14] Mallat S.G. A theory for multiresolution signal representation: the wavelet decomposition, IEEE Trans Pattern Anal Mach Intell 11, 7 (1989), 674-693.
[15] Bhaskaran V., and Konstantinides K. Image and video compression standards, algorithms and architectures. Kluwer Academic Publishers, Norwell, Massachusetts USA, 1995.

[16] Manjunath B.S., and Ma W.Y. Texture features for browsing and retrieval of image data, IEEE Trans Pattern Anal Mach Intell 18, 8 (1996), 837-841.

[17] Daugman J.G. Two-Dimensional Spectral Analysis of Cortical Receptive Field Profile. Vision Res 20, (1980), 847856.

[18] Daugman J.G. Uncertainty Relation for Resolution in Space, Spatial Frequency, and Orientation Optimized by TwoDimensional Visual Cortical Filters. J Optical Soc Amer, 2, 7 (1985), 1160-1169.

[19] Zhang D.S., Wong A.W., Indrawan M., and Lu G. Contentbased Image Retrieval Using Gabor Texture Features. In: Proceedings of the IEEE Pacifin-Rim international conference on multimedia, (2000), 392-395.

[20] Castelli V., and Bergman L. D. Image Databases: Search and Retrieval of Digital Imagery. New York, John Wiley \& Sons, 2002.

[21] Swain M.J., and Ballard D.H. Color indexing. Int J Comput Vis, 7, 1 (1991), 11-32.

[22] Manjunath BS., Ohm J.R., Vasudevan V.V., and Yamada A. Color and Texture Descriptors. IEEE Trans Cir Sys Vid Tech, 11, 6 (2001), 703-715.

[23] Laskaris N., and Ioannides A. Exploratory data analysis of evoked response single trials based on minimal spanning tree, Clinical Neurophysiology 112, (2001) 698-712.

[24] Costa J., and Hero A.O. Geodesic entropic graphs for dimension and entropy estimation in manifold learning. IEEE Trans Signal Process, 52, 8 (2004) , 2210-2221.

[25] Theoharatos C., Laskaris N.A., Economou G., and Fotopoulos S., Combining self-organizing neural nets with multivariate statistics for efficient color image retrieval, Comput. Vision Image Understand, 102, 13 (2006), 250-258.

[26] Laskaris N.A., and Fotopoulos S. A novel training scheme for Neural-Network based Vector Quantizers and its application in Image Compression, Neurocomput, 61, (2004), 421-427.

[27] Jain A.K., and Farrokhnia F. Unsupervised texture segmentation using Gabor filters. Pattern Recognit, 24, 12 (1991), 1167-1186.

[28] Manjunath B.S., and Chellappa R. Unsupervised texture segmentation using Markov random fields models. IEEE Trans Pattern Anal Mach Intell, 13, 5 (1991), 478-482.

[29] Ng I., Kittler J., and Illingworth J. Supervised segmentation using a multiresolution data representation. Signal Process, 3, 2 (1993), 133-163.

[30] Porter R., and Canagarajah N. A robust automatic clustering scheme for image segmentation using wavelets. IEEE Trans Image Process, 5, 4 (1996), 662-665.

[31] Liu Y., Zhang D., Lu G., and Ma W.Y. A survey of contentbased image retrieval with high-level semantics. Pattern Recognit, 40, 1 (2007), 262-282. 